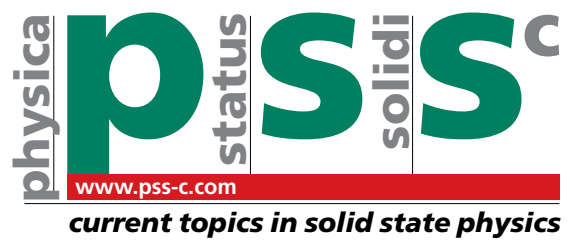

\title{
Cell proliferation monitoring by multiplexed electrochemical impedance spectroscopy on microwell assays
}

\author{
Stijn Duchateau ${ }^{*}, 1,2$, Jeroen Broeders ${ }^{1,2}$, Dieter Croux ${ }^{2}$, Daniel Janssen ${ }^{3}$, Jean-Michel Rigo ${ }^{3}$, \\ Patrick Wagner $^{2,4}$, Ronald Thoelen ${ }^{1,2}$, and Ward De Ceuninck ${ }^{2,4}$ \\ ${ }^{1}$ Xios University College, Agoralaan - Gebouw H, 3590 Diepenbeek, Belgium \\ ${ }^{2}$ Hasselt University, Institute for Materials Research, Wetenschapspark 1, 3590 Diepenbeek, Belgium \\ ${ }^{3}$ Hasselt University, Biomedical Institute, Agoralaan - Gebouw D, 3590 Diepenbeek, Belgium \\ ${ }^{4}$ IMEC - Division IMOMEC, Wetenschapspark 1, 3590 Diepenbeek, Belgium
}

Received 24 September 2012, revised 28 February 2013, accepted 1 March 2013

Published online 12 April 2013

Keywords impedance spectroscopy, cell proliferation, BV2, HEK-293, CHO

* Corresponding author: e-mail stijn.duchateau@uhasselt.be, Phone: +32 011268 818, Fax: +32 011268899

Cell proliferation can be monitored by a wide range of well-established techniques. Most of the principles used rely on optical, single end-point methods, often involving the use of absorbent, fluorescent or luminescent compounds. These additives can interfere with the cell growth, thus producing distorted results. Electrochemical impedance spectroscopy provides a solution for this problem and enables continuous monitoring without interference. However equipment for this measurement technique is often bulky, highly expensive and lacks multichannel features. This paper presents a low-cost, compact hardware platform optimized for proliferation measurements, together with custom software to ease interpretation and physical modelling of data. Performance is demonstrated and measurement parameters are fine-tuned for three commonly used cell types in proliferation measurements.
1 Introduction Common day proliferation tests are mostly optical single end-point techniques $[1,2]$. The use of absorbance, fluorescence or luminescence compounds necessary for these methods can severely interfere with the cell growth, which can affect the monitoring [3, 4]. Electrochemical impedance spectroscopy can provide an outcome for this problem. This label-free, non-invasive technique enables continuous monitoring of distinct behavior associated with the cell cycle. Previous examples include proliferation measurements on lymphocytes and leukemia cells $[5,6]$ and ex vivo monitoring of cancer cell behavior [7]. Furthermore, this technique can be used in the readout of living cell based biosensors [8] and has possible applications in the detection of cancer cells $[9,10]$.

Impedance spectroscopy allows for analysis of materials in an A.C. circuit by monitoring the complex ratio of the voltage to the current at different frequencies. This allows the interpretation of a biochemical system by means of equivalent electrical components $[11,12]$. Biological cells have a highly resistive membrane; thus when using a low measurement voltage the electric field will not go through the cell but will be disrupted. When measuring cells in a conductive fluid this results in a disturbance in the electric field and a resulting impedance change [13]. Since an increase or decrease in the surface coverage in a certain well will result in a change in conductivity at a certain excitation frequency it is possible to monitor cell proliferation by evaluating the impedimetric response. These measurements are often performed with specialized equipment. However, the basic measurement principles differ only slightly from general-purpose impedance spectroscopy units. This article discusses the steps necessary to make generally applicable impedance spectroscopy devices suitable for these measurements. First a miniature multiplexer is developed in which commercially available well plates, commonly used for proliferation measurements, can be mounted. Crucial excitation signal parameters are finetuned to give a maximized, accurate response for three 
specific cell types: Human embryonic kidney (HEK), Chinese hamster ovary $(\mathrm{CHO})$, and microglial BV2 cells. These all have in common that they are easy to grow in vitro and can be used in various research applications [14, 15]. Furthermore, physical properties of wells containing different cell types are characterized by equivalent circuit modeling. Time resolved measurements are used for the analysis of the electrode coverage which can serve as an indication of cell dynamics. All data analysis is performed with custom software.

\section{Materials and methods}

2.1 Impedance spectroscopy Previous research resulted in the development of a miniaturized eight-channel impedance analyzer [16], which is used as a starting point for the proliferation measurements. This eight-channel device is fine-tuned for biosensor measurements and is capable of measuring impedance from $10 \Omega$ to $5 \mathrm{M} \Omega$ in a frequency range from $10 \mathrm{~Hz}$ to $100 \mathrm{kHz}$. However, when working with living cells several additional design factors come into play.

In order to rule out any influence of the excitation signal on the cells, the excitation voltages should be carefully chosen. Typical resting potentials of animal cells lay around $70 \mathrm{mV}$ D.C. with the interior of the cell being negatively polarized with respect to the environment. When this voltage change reaches a threshold voltage, i.e. becomes less negative, it may trigger ion channels resulting in the release of certain ions [17]. These ions will thus enter the medium during measurements, altering the conductivity and the measured impedance. Furthermore, the release of these $\mathrm{Ca}^{2+}, \mathrm{Cl}^{-}$and $\mathrm{K}^{+}$ions can have a significant influence on the cell proliferation itself $[18,19]$. To prevent these ion releases from occurring, the excitation voltage of the device was set to $50 \mathrm{mV}$ peak to peak by adapting the amplifier stage of the impedance setup. Any unwanted DC voltages present in the measurement range are decoupled by means of capacitors connected to the output of the impedance analyzer [16].

Commercially available devices for impedimetric proliferation measurements operate only at fixed frequencies, typically in the $10 \mathrm{kHz}-100 \mathrm{kHz}$ range [20-22]. Since different cell types have different sizes and shapes the choice was made to sweep a broader spectrum so ideal measurement frequencies could be determined for each of the three cell types. To minimize cell excitation the measurement interval was set to 20 minutes with a single frequency sweep taking less than 5 seconds. Cell behavior can however also be influenced by low-frequency signals, as reported in [23]. Therefore, initially a frequency spectrum from $100 \mathrm{~Hz}$ to $100 \mathrm{kHz}$ was chosen. This spectrum is used to determine several physical properties of the cell types.

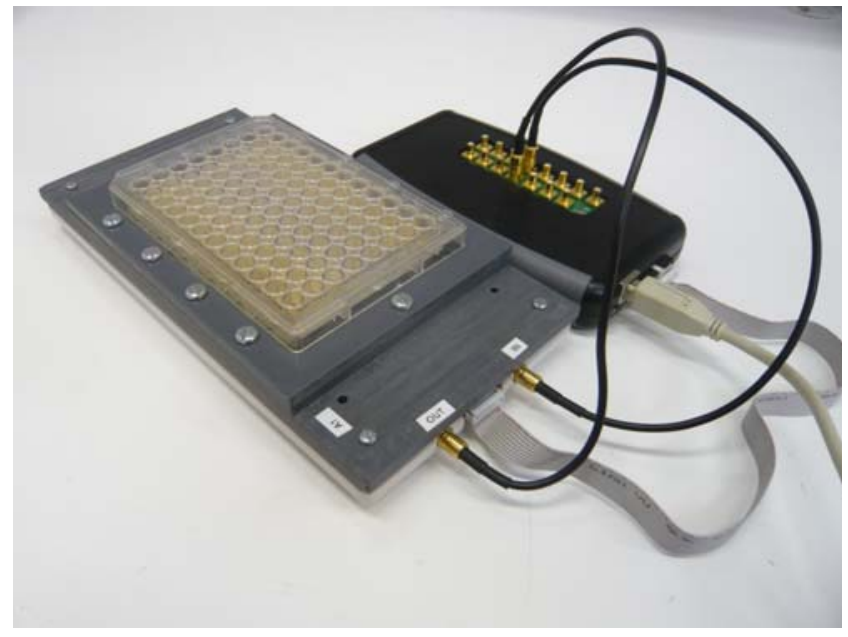

Figure 1 The impedimetric measurement setup, showing on the left side the 96 well plate on top of the multiplexer and on the right the miniature impedance analyser with built in calibration system.

2.2 Miniature multiplexer Cell proliferation measurements are commonly performed in microwell plates with standardized dimensions. In order to perform electrochemical impedance spectroscopy, electrodes need to be present inside each well. A Roche E-Plate with interdigitated gold electrodes was chosen for this purpose [24]. These E-plates consist of six glass substrates with 16 interdigitated structures each, fixed at the bottom. Each structure has an outer dimension of $6.94 \mathrm{~mm}^{2}$ with $80 \mu \mathrm{m}$ finger electrodes. To connect the impedance analyzer with the well plate and to switch between wells, a 96-channel multiplexer was developed. This device together with the miniature impedance analyser can be seen in Fig. 1. The design is focused on low internal losses and uses matched lines between individual channels. The compact size of the setup allows easy placement inside incubation ovens. It is possible, for high-throughput measurements to connect up to 8 multiplexers to 1 impedance analyser and perform a quasi-simultaneous measurement on 768 wells.

The electrical connection with these electrodes is made by means of spring contacts (Interconnect Devices Inc., UK). Channel switching to the different wells is done with miniature relays (Omron, UK) to keep the total size of the device as small as possible. These relays are controlled by Texas Instruments PCA9535 $\mathrm{I}^{2} \mathrm{C} \mathrm{I} / \mathrm{O}$ expanders [25]. This allows for control of the relays and thus well selection by means of a single $\mathrm{I}^{2} \mathrm{C}$ bus. This bus protocol is interfaced directly to the impedance analyser discussed in the previous paragraph. A single USB-port controller is used to control the miniature impedance analyser and multiplexer. This USB-port also provides power to the system, avoiding the need for any external power supply units. Measurements are performed by continuous switching between the user-selectable wells of the multiplexer unit. To avoid interference between these wells a switching delay of $100 \mathrm{~ms}$ was implemented. 
2.3 Data modeling Using Electrochemical Impedance Spectroscopy (EIS) to evaluate cell proliferation not only yields information on differences in the cell quantities inside a well. Using the right modelling tools it is even possible to evaluate the growth, the cell migration and changes in the interaction between the medium and the electrode surface. Custom software was developed to meet this demand. This software can generate Bode- and Nyquist plots, which are useful to select the optimal measurement frequency for the evaluation of cell proliferation. It is also possible to monitor this frequency over time. This is an essential tool in the examination of cell proliferation since changes in cell morphology could occur over time. The software also allows for a more in-depth analysis, using a fit on the time data to determine growth parameters such as the growth speed. A last functionality is added to find the equivalent electrical circuit parameters and to monitor their changes over time. A Levenberg-Marquardt algorithm [26] is used on the real part of the impedance to calculate the values of the components. The starting values for the algorithm are calculated based on the measurement data.

2.4 Cell cultures Murine derived BV-2 cells, CHO cells and HEK293T cells were cultured in culture flasks (NUNC, Roskilde, Denmark) with Dulbecco's Modified Eagle's Medium (DMEM), L-glutamine and high glucose (4500 $\mathrm{mg} / \mathrm{ml}$ ) without pyruvate (Invitrogen, Merelbeke, Belgium) supplemented with $10 \%$ fetal calf serum (FCS) and 100 $\mathrm{U} / \mathrm{ml}$ penicillin and $100 \mu \mathrm{g} / \mathrm{ml}$ streptomycin $(1 \% \mathrm{P} / \mathrm{S})$. The cells were incubated in a humidified incubator $(5 \% \mathrm{CO} 2)$ at $37^{\circ} \mathrm{C}$. After loosening the cells mechanically, the resulting cell suspension was centrifuged for 10 minutes at 800 rpm with a Jouan B3.11 (Thermo electron corporation, Waltham, MA, USA) and the cell pellet was re-suspended in fresh cell growth medium (DMEM). Subsequently, cells were seeded on a Roche E-Plate 96 for quantification of cell proliferation.

3 Results and discussion The E-Plate has a different base impedance for each row and a small deviation between wells on the same row due to differing lengths of the leads running to the well. It is therefore important to scale the measurements to make a precise comparison between the different concentrations of cells. The measured resistivity of these leads is either $35 \Omega, 55 \Omega, 80 \Omega$ or $105 \Omega$, with $\pm 5 \Omega$ accuracy, depending on the placement of the well. This is consistent with data provided by the manufacturer [20]. These offset values are taken into account for each discussed measurement.

3.1 Impedance spectrum Bode plots were generated to gain insight in the behaviour of each of the three analysed cell types. An amount of 30000 cells/well of respectively BV2, HEK and CHO cells was seeded with $100 \mu \mathrm{l}$ of DMEM medium into the wells. To rule out resistivity difference between measurements the DMEM medium was identical for all measurements discussed in this work. Figure 2 shows the initial Bode plots after a stabilization

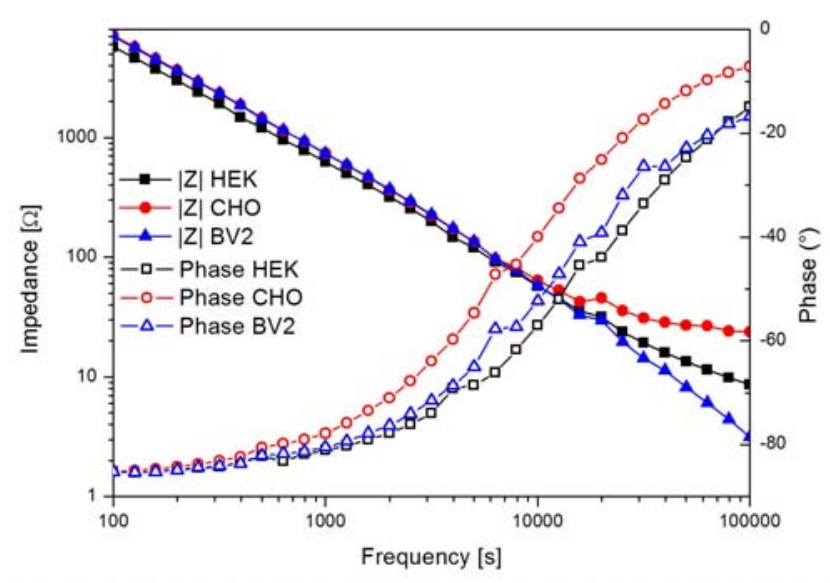

Figure 2 Bode plots for an amount of 30000 BV2, HEK and $\mathrm{CHO}$ cells after stabilisation at the start of the time measurement.

period of 20 minutes. In the low frequency range, $100 \mathrm{~Hz}$ to $10 \mathrm{kHz}$, the impedance drops for the three cell types under study. This corresponds to the typical phase shift of capacitive components. At higher frequencies the phase goes towards zero which makes it possible to distinguish individual components with the developed software. In the high frequency region of the Bode plot a differentiation in the impedance magnitude between the 3 cell types can be made due to differences in the surface coverage. When measuring an equal amount of CHO $(15 \mu \mathrm{m})$, HEK (13 $\mu \mathrm{m})$, and BV2 cells $(8 \mu \mathrm{m})$ the $\mathrm{CHO}$ cells will, because of a larger double layer interaction caused by a higher surface coverage, show a higher impedance.

An equivalent electrical circuit for a well plate with cell growth on top of the electrodes can be found in Fig. 3. The electrodes and the liquid commonly behave like pure resistors, as do present connectors and wires leading to the electrochemical cell. These are all summed together in the serial resistor $R_{S}$. In electrochemical systems a parallel circuit consisting of the double-layer capacitance $\mathrm{Q}_{\mathrm{dl}}$ and resistance $R_{d l}$ can be seen at the interface between a solid material and conducting liquid [27]. The electrical equivalent circuit of a living cell can also be reduced to a parallel $\mathrm{Q}_{\mathrm{C}}-\mathrm{R}_{\mathrm{C}}$ circuit. The influence of the cell resistance $\mathrm{R}_{\mathrm{C}}$ and the cell capacitance $\mathrm{Q}_{\mathrm{C}}$ cannot, due to the limited frequency window $(100 \mathrm{~Hz}$ to $100 \mathrm{kHz})$, be discerned from Fig. 3 and can thus be neglected in the equivalent circuit model. The remaining parallel R-RQ circuit is usually referred to as a Randles circuits [28]. Due to the compact size of the multiplexer on which the well plates are placed, the effect of lead and connector resistances can also be ignored. It can thus be concluded that the changes in magnitude of the impedance measured in Fig. 2 is primarily related to double layer $\left(\mathrm{R}_{\mathrm{dl}}\right)$ and liquid resistivity $(\mathrm{RL})$. The Levenberg-Marquardt fitting algorithm, discussed in the previous section, uses this reduced circuit of Fig. 3 (R-RQ) to calculate the equivalent measurement model. 

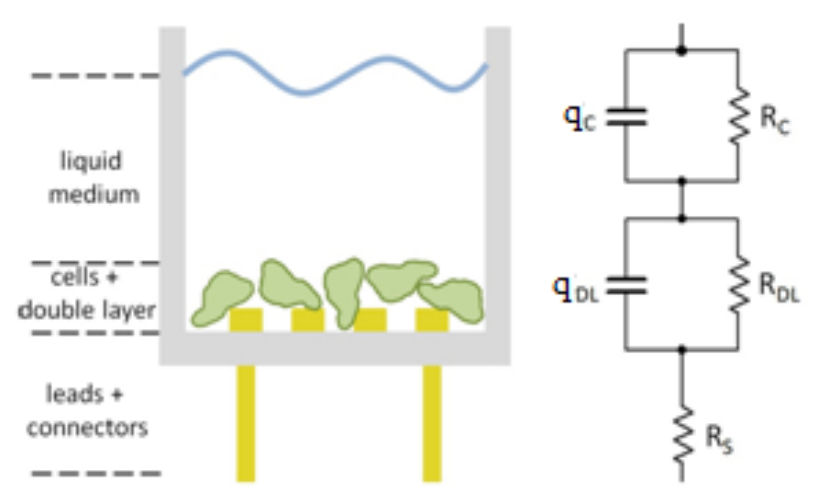

Figure 3 Schematic side view of a measurement well together with the electric equivalent circuits for wells populated by the three cell types.

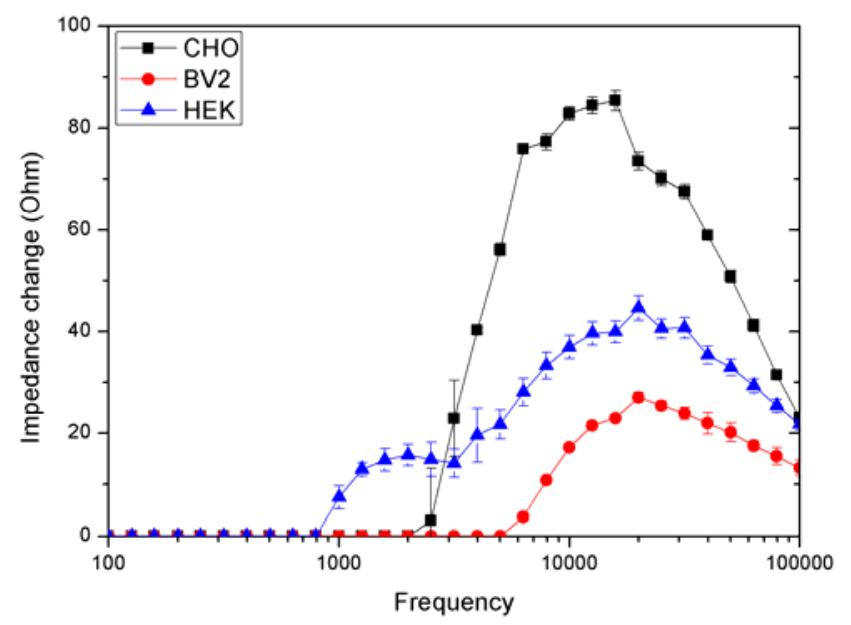

Figure 4 Average of 8 measurements of the maximum impedimetric response over $100 \mathrm{~h}$ versus frequency.

For the frequency window starting at 100 to $1000 \mathrm{~Hz}$ Eq. (1) can be deducted to calculate the real and the imaginary part of the impedance. The starting values for the algorithm are determined from the measurement data. As the influence of the parallel circuit of the double-layer is negligible at higher frequencies $R_{s}$ is calculated at the point where the phase approximates zero. When in a parallel circuit the magnitude value of a component is more than 1000 larger than the other it can be discerned from calculation. At lower frequencies, the impedance of $\mathrm{Q}_{\mathrm{dl}}$ is much larger compared to the double layer resistance $\left(\mathrm{R}_{\mathrm{dl}}\right)$. The software uses the highest impedance at the start of the measurement minus the estimated value for $R_{s}$ as initialization value for $\mathrm{R}_{\mathrm{c}}$.

3.2 Ideal excitation frequency As was shown in the previous paragraph, there is a difference in the impedance magnitude of the cells in the high-frequency region. This change can be an indicator for the size of the cells present in the well.

$$
\begin{gathered}
\mathrm{Re}=\mathrm{R}_{\mathrm{s}}+\frac{\mathrm{R}_{\mathrm{dl}} \times\left(\frac{1}{\mathrm{Qdl}_{\mathrm{dl}} \times(2 \pi \mathrm{f})^{\mathrm{n}}}\right)^{2}}{\mathrm{R}_{\mathrm{dl}}{ }^{2}+\left(\frac{1}{\mathrm{Q}_{\mathrm{dl}} \times(2 \pi f)^{\mathrm{n}}}\right)^{2}} \\
\mathrm{Im}=\frac{\mathrm{R}_{\mathrm{dl}}^{2} \times \frac{1}{\mathrm{Q}_{\mathrm{dl}} \times(2 \pi f)^{n}}}{\mathrm{R}_{\mathrm{dl}}{ }^{2}+\left(\frac{1}{Q_{\mathrm{dl}} \times(2 \pi f)^{n}}\right)^{2}}
\end{gathered}
$$

Equation (1) Calculation of the Re and Im part of the impedance with $R_{s}$ the serial resistance, $R_{c}$ cell resistance and $\mathrm{Q}_{\mathrm{c}}$ cell capacitance.

Although it is possible to monitor the electrode coverage by exciting the wells with the whole frequency range, a single ideal excitation frequency can be determined to achieve the most distinct results and the most accurate monitoring of proliferation. To determine this ideal working frequency, the maximal impedimetric response was recorded for each measured frequency for the three cell types. An amount of 30000 cells/well of respectively BV2, HEK and $\mathrm{CHO}$ cells was measured twice in quadruplets for 100 hours. Figure 4 shows the maximal response between 1 $\mathrm{kHz}$ and $100 \mathrm{kHz}$ during the measurement time. The BV2 and HEK cells will reach a maximmum at $20 \mathrm{kHz}$, while the highest response for the $\mathrm{CHO}$ cells is at $15 \mathrm{kHz}$. This is likely due to the higher impedance of the $\mathrm{CHO}$ cells. Most commercially available equipment will only evaluate a few frequencies between 10 and $100 \mathrm{kHz}$ and take the average impedance change over this range and will thus have a reduced sensitivity for cell changes. This shows the advantage of performing a frequency sweep where the ideal frequency can be found and the detection limit can be optimized.

3.3 Proliferation monitoring To illustrate the practical usability of this impedimetric measurement technique, different amounts of BV2, HEK and CHO cells were continuously monitored over a time interval of 100 hours. Amounts of 1000, 3000, 6000, 10000 and 30000 BV2, $\mathrm{HEK}$ and $\mathrm{CHO}$ cells/well were seeded, together with reference channels only containing culture medium. These reference channel are used to rule out external effects, e.g. temperature fluctuations, which would affect the absolute impedance values [29]. The average impedance of the wells versus the measurement time is given in Fig. 5. The measured impedance of the reference channel is subtracted from all channels. Measurements were performed at an excitation frequency of $20 \mathrm{kHz}$ for BV2 and HEK cells and $15 \mathrm{kHz}$ for $\mathrm{CHO}$ cells, as was determined in the previous paragraph. Since the measured impedance is related to electrode-cell interaction, one can see the evolution of population size for each concentration of seeded cells. 

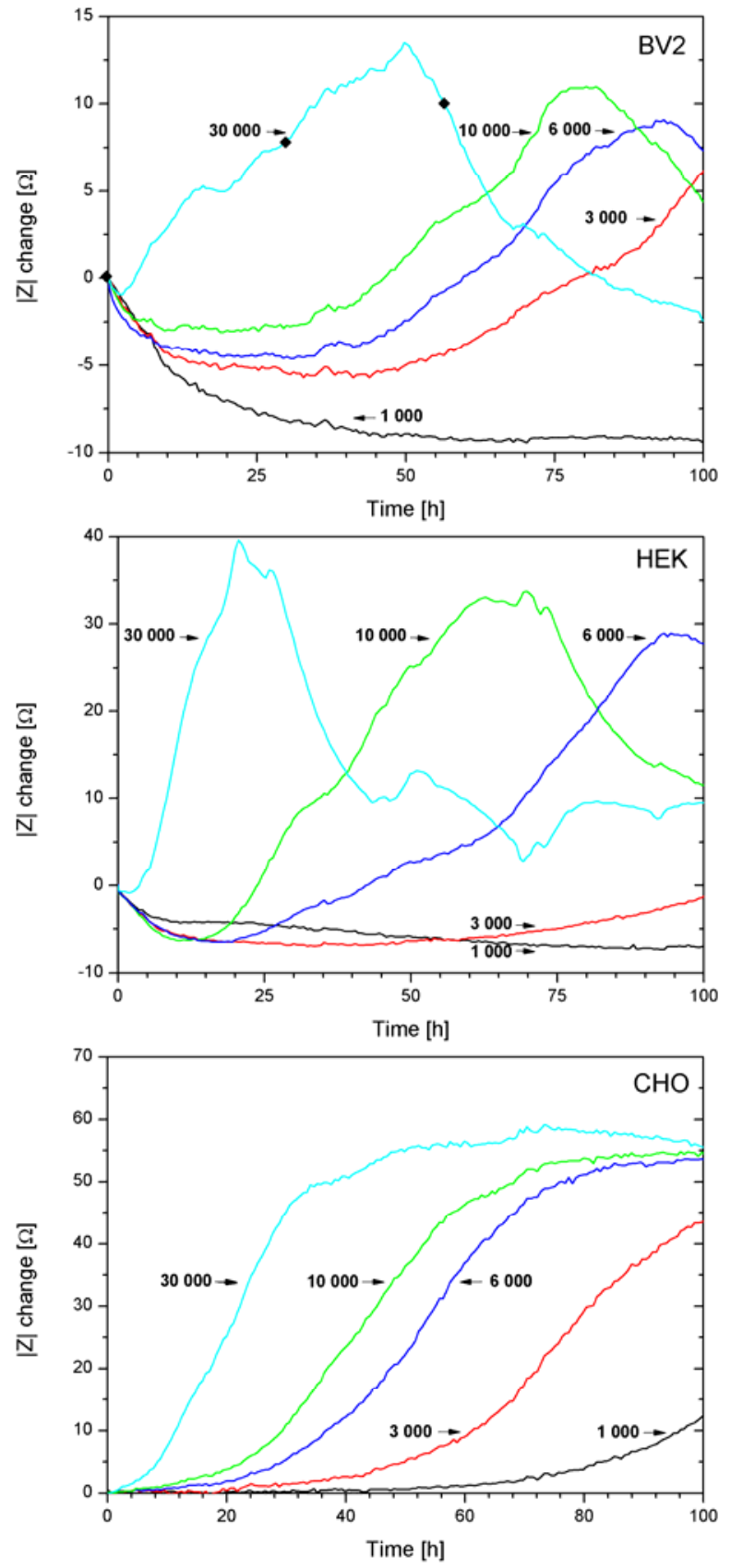

Figure 5 Impedimetric monitoring of the cell proliferation for different concentrations of BV2 (a), HEK (b) and CHO (c).

The impedance change over time progresses differently for each cell type. The evolution and magnitude of this change depends on two factors, the size of the cells and their doubling time. BV2 doubles in approximately $15 \mathrm{~h}$ while HEK $(24 \mathrm{~h})$ and $\mathrm{CHO}(14-17 \mathrm{~h})$ need more time to grow [30]. The monitoring of this dynamic evolution of growth can be advantageous for e.g. drug tests. For drug testing purposes classical end point methods, for example an absorption test [31] or an enzyme-linked immunosorbent assay (ELISA) only yields static data [32]. This cannot give a clear indica- tion of the number of cells present in the setup. In such tests, EIS can be used to get an indication of the number of available cells, the cell type and the growth stage.

Since the starting impedance of the interdigitated structures at the wells can be determined, it is possible to have an indication of the number of cells present. When knowing the cell line, the magnitude of the impedance change and the measurement duration the graphs can be used to get an indication of the number of cells at the start of the measurement. If the doubling time of the cells in the specific medium is known and the start value would be multiplied with this factor a rough estimate for the number of cells present in the well can be found.

If on the other hand only the measurement time and the starting amount of cells is known, the evolution of the graphs can also be used to determine the cell type under test. For these purpose it is evident that a gross number of cells needs to be quantified with this technique in order to find the right growth curve. The three discussed types all have different growth curves, which are reconfirmed over multiple measurements at different times and at different locations on the well plate, which show distinct differences during the measurement time. For example in Fig. 5(a), a drop in impedance is noticed on all wells containing a population of BV2 cells. This drop during the stabilization phase is most likely due to nesting of the cells on the electrodes. After this stabilization, an increasing impedance is observed on the wells containing the relatively high amount of 30000 cells. The wells containing lower amounts need more time to start their growth phase. The impedance reaches a distinct maximum after a certain time interval, when the maximum measurable population size is reached. After this maximum, impedance decreases almost exponentially and the medium changes from the normal pink to a darker yellow colour. This is most likely caused by cell death which can be initiated by, among others, necrosis, apoptosis, or autophagy. Further research is needed to make a decisive conclusion. Figure 6 shows pictures taken with the Zeiss Axiovert 100 to verify these findings. Figure 6(a) shows the 30000 BV2 cells directly after seeding. The cells are spread all across the surface of the well and there is a large distance between individual cells. $30 \mathrm{~h}$ later, in Fig. 6(b) the cells have already more than doubled their numbers and the cells are still growing. Figure $6(\mathrm{c})$ shows the start of the cell death stage. In the centre of the cells little black dots start to occur which indicates cell death. The HEK cells in Fig. 5(b) exhibit a similar behaviour as the BV2 cells, though these cells reach a maximum measurable population earlier, which results in a steeper rise time. The maximum $\mathrm{CHO}$ population is reached after a similar time as the BV2 population, this population size is maintained much longer. 


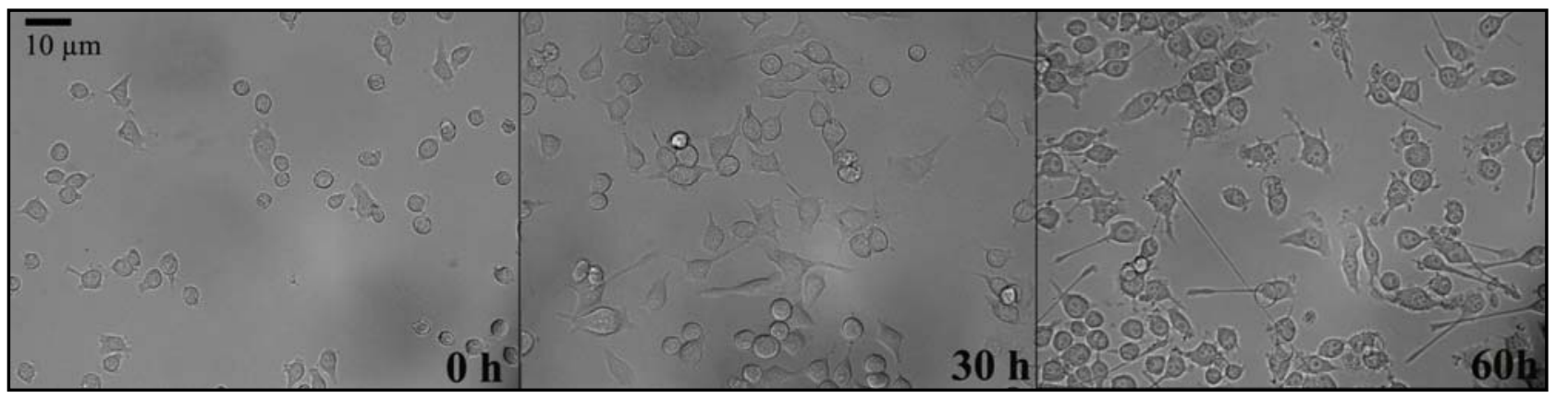

Figure 6 Evolution of BV2 cell growth at $0 \mathrm{~h}, 30 \mathrm{~h}$ and $60 \mathrm{~h}$.

Table 1 Values for the equivalent circuit at the start, end and maximum of the impedance measurement for the 3 cell types

\begin{tabular}{|c|c|c|c|c|c|c|}
\hline & & $R_{\mathrm{S}}(\Omega)$ & $R_{\mathrm{dl}}(\Omega)$ & $\begin{array}{l}Q_{\mathrm{dl}} \\
(\mu \mathrm{F})\end{array}$ & $n$ & $R^{2}$ \\
\hline \multirow[b]{3}{*}{$\mathrm{CHO}$} & $T_{0}$ & 103 & 53911 & 13.12 & 0.42 & 1 \\
\hline & $T_{\max }$ & 165 & 362899 & 0.67 & 0.64 & 0.99 \\
\hline & $T_{100}$ & 165 & 286791 & $0 ., 56$ & 0.68 & 0.99 \\
\hline \multirow[b]{3}{*}{ HEK } & $T_{0}$ & 43 & 51726,4 & 15.3 & 0.45 & 0.97 \\
\hline & $T_{\max }$ & 79 & 71834 & 18.8 & 0.44 & 0.99 \\
\hline & $T_{100}$ & 66 & 115398 & 0.68 & 0.73 & 1 \\
\hline \multirow[b]{3}{*}{ BV2 } & $T_{0}$ & 63 & 66427 & 10.8 & 0.43 & 0.99 \\
\hline & $T_{\max }$ & 76 & 79500 & 22.9 & 0.31 & 0.98 \\
\hline & $T_{100}$ & 70 & 108049 & 0.65 & 0.74 & 1 \\
\hline
\end{tabular}

Converting the measured impedance and phase to the equivalent circuit discussed in the previous chapter could, since the measured difference is caused by an increase or decrease in electrode coverage, yield some more insights in the cell evolution. Table 1 presents the values for this conversion using Eq. (1) at the start, maximum value and end of the measurement for an amount of 30.000 cells. A relatively small change, 17 to $43 \Omega$ could be seen in the series resistor of the three types of investigated cells. The double layer components, on the other hand, change more spectacular. The constant phase element value drops, for all cells, to approximately $0.5 \mu \mathrm{F}$. However, the parallel resistance of the different cells changes more diverse.

When the types of cells, the amount and measurement time are known than the state of the cells can be deduced from the graphs. This can be of interested for drug research, since some drugs might have a bigger impact at a different stage of cell growth.

4 Conclusions A novel hardware setup, consisting of a miniature impedance analyser and a 96-channel multiplexer is presented. Specific design aspects, such as the measurement voltage and frequencies were taken into account to allow non-invasive measurements on several types of biological cells. Custom software was implemented to facilitate data processing when continuously monitoring proliferation. The first measurements show the ability to measure impedance changes due to cell-electrode interaction for three commonly used cell types. The change was type specific which could indicate that, when working with a known amount of cells, the impedance can be used to give information on the diameter and thus the type of the cell.

In comparison to commercially available measurement setups it is possible to measure up to 768 wells quasisimultaneously, which makes the setup perfectly suited for high throughput measurements. Since the device can measure a large frequency spectrum it was possible to determine ideal excitation frequencies for the different cell types. These frequencies where used for the continuous monitoring of the cells. Measurements showed that the proliferation process was visible in the impedimetric response and that each cell type has it is own growth dynamics. These could be used to get an indication of the number of available cells, the cell type and the growth stage. The measurements also show that a larger starting concentration of cells reaches its maximum impedance, thus maximum measurable population before depletion of the medium, much faster than lower concentrations. Furthermore, the distinct differences in growth rate between the specific cell types can easily be monitored. When using these cell lines in research the growth rates and times have to be taken into account.

Impedance spectroscopy is a promising technique in cell proliferation measurements, as it allows for continuous monitoring of population sizes. This non-invasive technique will most likely be applied in, amongst others, the testing cell population response to different compounds in biomedical, pharmaceutical, and toxicological applications.

Acknowledgements The authors would like to express their gratitude towards the Technical Institute Heilig Hart in Hasselt, in particular to Rudy Brouwers and Danny Daelemans, for support with enclosure design. Furthermore, we owe thanks to Wouter Vanaken, Johnny Baccus, Lieven De Winter and Jan Mertens for technical advice and stimulating discussions. 


\section{References}

[1] X. Zhang and S.-T. Yang, Proc. Biochem. 46, 2030-2035 (2011).

[2] B. J. C. Quah and C. R. Parish, J. Immunol. Meth. 379, 1-14 (2012).

[3] D. P. Maia et al., Chem.-Biol. Interact. 180, 220-225 (2009).

[4] M. Kressel and P. Groscurth, Cell Tissue Res. 278, 549-556 (1994).

[5] P. Upadhyay and S. Bhaskar, J. Immunol. Meth. 244, 133137 (2000).

[6] L. Ding et al., Electrochem. Commun. 9, 953-958 (2007).

[7] Q. Liu et al., Europ. Neuropsychopharmacol. 19, 796-805 (2009).

[8] H. Chen et al., Food Chem. Toxicol. 50, 612-618 (2012).

[9] Y. Zou and Z. Guo, Med. Eng. Phys. 25, 79-90 (2003).

[10] R. Wang et al., Biomed. Chromatography 26, 393-399 (2012).

[11] S. E. Moulton et al., Electrochim. Acta 49, 4223-4230 (2004).

[12] L. Wang et al., Biosens. Bioelectron. 25, 990-995 (2010).

[13] P. Ellappan and R. Sundararajan, J. Electrostatics 63, 297307 (2005)

[14] F. Bollin et al., Protein Express. Purification 78, 61-68 (2011)
[15] J. Zhu, Biotechnol. Adv. 29, 40-53 (2011)

[16] J. Broeders et al., Phys. Status Solidi A 208, 1357-1363 (2011).

[17] C. H. Fry and R. I. Jabr, Surgery 28, 49-54 (2010)

[18] M. Sunagawa et al., J. Physiol. Sci. 60, S202-S202 (2010).

[19] C. Eder, Am. J. Physiol.-Cell Physiol. 275, C327-C342 (1998)

[20] E. Urcan et al., Dental Mater. 26, 51-58 (2010).

[21] H. Slanina et al., Int. J. Med. Microbiol. 301, 75-75 (2011).

[22] L. Ceriotti et al., Sens. Actuators B 123, 769-778 (2007).

[23] R. Tonini et al., Biophys. J. 81, 2580-2589 (2001).

[24] R. Diagnostics. E-Plate 96, available: www.roche-appliedscience.com/proddata/grip/3 $8 \begin{array}{lllllllll}3 & 2 & 1 & 0 . h t m l\end{array}$

[25] T.I. Inc., PCA9535 data sheet, available: http://www.ti.com/lit/ds/symlink/pca9535.pdf

[26] K. Levenberg, Quart. Appl. Math. 2, 164-168 (1944).

[27] N. Kallay et al., Croat. Chim. Acta 77, 243-249 (2004).

[28] F. Lisdat and D. Schafer, Analyt. Bioanalyt. Chem. 391, 1555-1567 (2008).

[29] B. van Grinsven et al., Phys. Status Solidi A 207, 919-923 (2010).

[30] L. standards, 2013, http://www.lgcstandards-atcc.org

[31] O. Mignen et al., J. Cell Sci. 118, 5615-23 (2005).

[32] J. X. Bi et al., Biotechnol. Bioeng. 85, 741-9 (2004). 